INPLASY

PROTOCOL

To cite: Huang et al. The Effects of Anodal Transcranial Direct Current Stimulation on Delaying Fatigue of Different Modes of Motion: A Metaanalysis. Inplasy protocol 202190104. doi:

10.37766/inplasy2021.9.0104

Received: 28 September 2021

Published: 28 September 2021

Corresponding author:

Xizhang Huang

215541311@qq.com

Author Affiliation:

Shanghai University of Sport

Support: Shanghai University of Sport.

Review Stage at time of this submission: Preliminary searches.

Conflicts of interest:

None declared.

\section{The Effects of Anodal Transcranial Direct Current Stimulation on Delaying Fatigue of Different Modes of Motion: A Meta-analysis}

\author{
Huang, X1; Gao, B²; Wang, G³.
}

Review question / Objective: The aim of this study is to analyze the intervention effect of transcranial direct current stimulation (tDCS) on delaying fatigue during exercise.

Rationale: tDCS is a kind of noninvasive brain stimulation techniques, it usually includes target electrode (anode) and the reference electrode (cathode), passed on to the brain is constant, the weak current (1-2 mA), stimulate the neurons resting membrane potential changes, to reach the brain cortex excitability, is a kind of regulation of non invasive brain cortex neuron function control technology.tDCS has positive effects in promoting motor skill learning, enhancing muscle explosive power, improving muscle endurance level and relieving skeletal muscle pain.This study intends to conduct a meta-analysis of tDCS intervention experiments in healthy people. Two outcome indicators, time to exhaustion test (TTE) and rating of perceived exertion (RPE), are selected to explore whether tDCS has an improvement effect on delaying fatigue under different exercise modes, and to explore whether different stimulation location, stimulation duration and current intensity have a positive effect on delaying fatigue.To provide evidence basis for the application of this method in sports practice.

INPLASY registration number: This protocol was registered with the International Platform of Registered Systematic Review and Meta-Analysis Protocols (INPLASY) on 28 September 2021 and was last updated on 28 September 2021 (registration number INPLASY202190104).

\section{INTRODUCTION}

Review question / Objective: The aim of this study is to analyze the intervention effect of transcranial direct current stimulation (tDCS) on delaying fatigue during exercise.

Rationale: tDCS is a kind of noninvasive brain stimulation techniques, it usually includes target electrode (anode) and the 
reference electrode (cathode), passed on to the brain is constant, the weak current (1-2 $\mathrm{mA})$, stimulate the neurons resting membrane potential changes, to reach the brain cortex excitability, is a kind of regulation of non invasive brain cortex neuron function control technology.tDCS has positive effects in promoting motor skill learning, enhancing muscle explosive power, improving muscle endurance level and relieving skeletal muscle pain.This study intends to conduct a meta-analysis of tDCS intervention experiments in healthy people. Two outcome indicators, time to exhaustion test (TTE) and rating of perceived exertion (RPE), are selected to explore whether tDCS has an improvement effect on delaying fatigue under different exercise modes, and to explore whether different stimulation location, stimulation duration and current intensity have a positive effect on delaying fatigue.To provide evidence basis for the application of this method in sports practice.

Condition being studied: According some studies reported, several studies have shown that tDCS can improve athletic performance, but some have claimed that tDCS does not significantly improve TTE or RPE during exhaustive exercise. Therefore, there is no further mechanism for the application of tDCS in sports performance.

\section{METHODS}

Search strategy: We selected relevant studies published until September 28, 2021, by searching PubMed (MEDLINE), Embase databases, Web of Science, Cochrane library. We applied no language restrictions.

Participant or population: Enrolled healthy adults.

Intervention: Anodal transcranial direct current stimulation.

Comparator: Sham stimulation.

Study designs to be included: No study restrictions.
Eligibility criteria: Newcastle-Ottawa Quality Assessment Scale (NOS) was used as a methodological quality assessment. Studies with a score equal to or higher than six were considered as high-quality studies.

Information sources: All intended information sources will come from electronic databases.

Main outcome(s): Time to exhaustion test (TTE) and rating of perceived exertion (RPE).

Additional outcome(s): None.

Data management: Two reviewers independently extracted the relevant data for each trial by using a standardized dataextraction form. Discrepancies were resolved through discussion or by a third reviewer. The extracted data included information regarding the source, country, participant characteristics, exercise protocol, intervention details, and outcome measures. The mean and standard deviation (SD) outcome data were extracted. For studies that met the inclusion criteria, if these values were not present, we attempted to contact the corresponding authors in order to obtain these values. In addition, we estimated raw data from graphs by using WebPlotDigitizer software (v4.2, San Francisco, CA, USA) (https://apps.automeris.io/wpd/), which were not presented in tables or text.

Quality assessment / Risk of bias analysis: Following the identification of the studies included within this review, the quality and risk of bias were assessed. This included a Cochrane risk of bias assessment tool to assess the risk of bias within the randomized controlled trials. The Cochrane risk of bias assessment tool evaluates randomized controlled trials based on several categories that include sequence generation, allocation concealment, blinding of participants and personnel, blinding of outcome assessment, incomplete outcome data, selective outcome reporting, and 'other issues.' Grades for these categories were provided 
as either 'high risk of bias', 'low risk of bias', or 'unclear risk of bias'.

Strategy of data synthesis: Data for each included trial were extracted by pairs of independent reviewers using a standardised data extraction form and discrepancies resolved through discussion. Extracted data included: study characteristics (eg, source, study design, country, participant's characteristics, outcome measure, description of the intervention/control groups and follow-up periods), means and measures of variability for all outcomes. When possible, raw mean and SD outcome data for both the intervention group and control group were extracted. We also estimated raw data from graphs in cases where this information was not presented in tables or text. We attempted to contact authors of included RCTs to clarify any relevant information or request additional data when required. To measure the intervention effect on continuous outconmes, we calculated the mean difference (MD) and 95\% condidence interval $(95 \% \mathrm{Cl})$. The $\mathrm{MD}$ and $95 \% \mathrm{Cl}$ weighted by the inverse variance method was measured using a random-effects model. Heterogeneity was assessed using $X^{2}(p 75 \%)$, as well as the visual inspection of the forest plot. All analyses were performed using Review Manager 5.3 (Copenhagen: The Noedic Cochrane Centre). If conversion was not possible due to the nature of outcome (eg, categorical or ordinal), we did not convert the results but instead presented them as a narrative synthesis.

Subgroup analysis: If necessary, we will analyze different stimulation area, stimulation duration, and current intensity between Anodal tDCS and Sham on the outcomes of TTE and RPE.

Sensitivity analysis: Sensitivity analysis was conducted mainly by changing the inclusion criteria.

Language: English.

Country(ies) involved: China.
Other relevant information: None.

Keywords: transcranial direct current stimulation; fatigue; time to exhaustion test; the rate of perceived exertion; metaanalysis.

Contributions of each author:

Author 1 - Xizhang Huang - The author retrieving documentation, extracting data, analyzing data and drafted the manuscript.

Email: 215541311@qq.com

Author 2 - Binghong Gao - The author retrieving documentation ,extracting data and analyzing data.

Email: gaobinghong@126.com

Author 3 - Gang Wang - The author provided feedback and approved the final manuscript.

Email: wg68@163.com 